\title{
A Numerical Investigation of the Resin Flow Front Tracking Applied to the RTM Process
}

\author{
Jeferson Avila Souzaa*, Luiz Alberto Oliveira Rocha, \\ Sandro Campos Amico ${ }^{\mathrm{b}}$, José Viriato Coelho Vargas ${ }^{\mathrm{c}}$ \\ ${ }^{a}$ Escola de Engenharia, Universidade Federal de Rio Grande - FURG, \\ Av. Itália, Km 08, s/n, Campus Carreiros, CEP 96201-900, Rio Grande, RS, Brazil \\ ${ }^{\mathrm{b}}$ Departamento de Engenharia de Materiais, \\ Universidade Federal do Rio Grande do Sul - UFRGS, \\ Av. Bento Gonçalves, 9500, CEP 91501-970, Porto Alegre, RS, Brazil \\ 'Departamento de Engenharia Mecânica, Universidade Federal do Paraná - UFPR, \\ CP 19011, CEP 81531-990, Curitiba, PR, Brazil
}

Received: March 16, 2011; Revised: May 31, 2011

\begin{abstract}
Resin Transfer Molding (RTM) is largely used for the manufacturing of high-quality composite components and the key stage during processing is the resin infiltration. The complete understanding of this phenomenon is of utmost importance for efficient mold construction and the fast production of high quality components. This paper investigates the resin flow phenomenon within the mold. A computational application was developed to track the resin flow-front position, which uses a finite volume method to determine the pressure field and a FAN (Flow Analysis Network) technique to track the flow front. The mass conservation problem observed with traditional FE-CV (Finite Element-Control Volume) methods is also investigated and the use of a finite volume method to minimize this inconsistency is proposed. Three proposed case studies are used to validate the methodology by direct comparison with analytical and a commercial software solutions. The results show that the proposed methodology is highly efficient to determine the resin flow front, showing an improvement regarding mass conservation across volumes.
\end{abstract}

Keywords: RTM, resin transfer, finite volume, flow front tracking, FAN

\section{Introduction}

The use of composites materials has increased worldwide since the 50 s, being mostly considered an alternative to traditional metals. Many processes have been developed for the manufacturing of composite materials. Among these techniques, RTM (Resin Transfer Molding) is regarded as fast, flexible and capable of producing parts with good surface finishing on both sides. In the RTM process, a dry fibrous preform is placed in the mold cavity, being subsequently impregnated with a liquid resin. The resin enters the mold through a number of injection ports (holes) and slowly flows within the mold cavity.

The numerical problem comprises the determination of the resin advancement inside the mold cavity. Knowledge of the shape and position of the resin/air interface as a function of the injection time is usually the main goal of the simulation. With this information, the mold designer is able to determine the optimum position of inlet and outlet gates. An adequate mold design should minimize injection time, avoid resin wasting through unnecessary output holes and allow the production of a homogeneous (density, resin content, void fraction, among others) final part. These characteristics could also be achieved following a trial-and-error experimental procedure, but this is highly expensive and time consuming.

The fibrous reinforcement media is mathematically formulated as a porous media and Darcy's Law is used to correlate pressure field and velocity inside the computational domain. Published works show that the available mathematical models considerably differ in complexity. In these models, the medium is normally considered homogeneous regarding porosity, but they may be isotropic ${ }^{1}$, i.e. with constant permeability in all directions, or non-isotropic ${ }^{2,3}$, when permeability is set as a tensor. In some experimental works, porosity of the fibrous media is also considered to vary (e.g. as a function of the medium) in the empirical mathematical formulation ${ }^{4}$. In addition, the model can be isothermal or include the temperature field determination inside the mold. For non-isothermal models, resin viscosity is formulated as a function of the temperature and time, and this also allows the investigation of the resin curing process ${ }^{5}$.

For isothermal problems, which is the focus of the present work, the resin advance transport phenomenon in RTM problems is usually solved in two steps: i) determination of the pressure field inside the computational domain filled with resin, and ii) determination of the resin flow-front line (interface between resin and air). For the first, as shown later, it is necessary to solve a second order Laplace equation for the pressure. This is normally an easy task, although difficulties related to discretization of complex geometries may arise. In the great majority of the reported works, the referred Laplace equation is solved using a finite element method, which is the obvious choice for complex geometry discretized domains. The difficulty associated with the use of the Finite Element method regards the evaluation of the mass flow-rate along the grid, which is commonly carried out by creating control volumes around the grid nodes and using the element local system of coordinates to calculate the resin fluxes along the 
faces of these control volumes. This particular method is called the FE-CV (Finite Element-Control Volume) method ${ }^{6,7}$.

The finite difference method is rarely used to determine the pressure field in RTM problems. For simple geometries, the finite difference method is much easier to formulate and program than the finite element method. On the other hand, if a complex geometry must be discretized, the computational implementation using the former method may become as complex as that using the latter.

Finite volume methods can also be used to determine the pressure field inside the computational domain ${ }^{3}$. They have long been associated with incompressible flows and simple geometry discretization perhaps due to the fact that earlier works focused on the treatment of the non-linear terms of the momentum equations, paying less attention to geometry discretization ${ }^{8}$. Indeed, the first formulations ${ }^{9}$ were difficult to implement for complex geometries, but the "new generation" of methods are fully capable of being used with unstructured grids for both $2 \mathrm{D}^{10,11}$ and $3 \mathrm{D}$ geometries ${ }^{12}$.

In this work, a finite volume method is used to determine the pressure field inside an RTM mold. The advantage of using this method in comparison with FE-CV methods is that the latter presents difficulties related to mass conservation of the resin flow along the grid elements. According to Joshi et al..$^{13}$, mass conservation is sometimes violated when the Galerking formulation is used with isoparametric finite elements to calculate the pressure field. The authors observed, for rectilinear (1D) flows, that the mass conservation was never violated, whereas for $2 \mathrm{D}$ flows, the error could reach $10 \%$. Others authors have also reported on the mass conservation problem associated with the FE-CV methods ${ }^{14,15,17}$.

On the other hand, conservation of all properties (e.g. mass, momentum, energy) is already incorporated into the basic formulation of the finite volume methods $s^{8,9,16}$, allowing easy calculation of the mass flow rate through the faces of all grid volumes. In the particular case of resin flow in a porous media where Darcy's equation is used to calculate flow velocity, the pressure equation to be solved is actually the continuity equation for an incompressible fluid in which velocity is written as a function of the pressure and the physical properties of the resin and the media. Since a finite volume method has been used to solve this equation, mass conservation across the grid is automatically enforced for the volumes fully filled with resin. Furthermore, a procedure, described in section 2.3, is proposed to approximate mass balances for the flow front volumes.

The position of the resin front line is determined by monitoring volume faces that connect empty volumes (resin volume fraction equal to zero) to volumes that are partially (or fully) filled with resin ${ }^{17}$. The front line volumes are usually only partially filled and the resin position is actually somewhere inside that control volume, instead of the control volume boundaries predicted by the mentioned algorithm ${ }^{17}$. All published works in the literature focusing on the FE-CV method applied to RTM problems use a similar front line determination algorithm. On the contrary, geometry reconstruction algorithms are usually employed with the VOF (Volume of Fluid) method ${ }^{18}$.

VOF is a general method largely used to model multiphase flows with two or more inviscid fluids which has been sometimes applied to track the resin flow front position in RTM problems ${ }^{19,20,21}$. This technique requires larger computational effort because a system of differential equations needs to be solved. The solution model includes momentum, continuity and volume fraction differential equations which are simultaneously solved in time and space. One of the advantages of this method relies on the fact that it can be applied to volumes with or without the solid phase (reinforced media), allowing, for example, to model the inlet channels of the RTM-Light process, a variant of the RTM process in which part of the flow occurs through peripheral open channels.
In VOF method the volume fraction $f$ of each fluid volume is determined by solving a transport equation for $f$, but this solution tends to smear in the volumes close to the moving surface ${ }^{22}$. The gradient of $f$ should be singular at the interface of two inviscid fluids, however it becomes finite in the numerical solution, causing this abnormality. This problem has been originally addressed using the donor-acceptor scheme ${ }^{18}$. This scheme identifies one cell as the donor of a particular amount of fluid from one phase and another (neighbor) cell as the acceptor of that amount of fluid, this in turn prevents numerical diffusion at the interface ${ }^{23}$. Several others algorithms, many of them highly complex, have been proposed to avoid numerical diffusion and oscillations ${ }^{19,24,25}$.

In the present work a new flow front monitoring technique is proposed. This approach differs from the VOF methods in which geometry reconstruction is part of the mass balances through the front line (resin/air) control volumes, and also differs from the FE-CV methods where the flow front is determined as the interface between empty elements and those with resin. The proposed technique uses a flow tracking algorithm to precisely determine the resin/air interface inside the grid elements. The aim of this algorithm is not to ensure mass conservation, what is intrinsic of the finite volume formulation, but to determine, even for a coarse grid, a realistic flow front position, allowing the geometrical reconstruction of the flow front geometry.

\section{Numerical Solution}

The computational domain shown in Figure 1 represents an actual lab-scale mold geometry. In this example, the injection hole is located at the center of the mold and the radial flow of the resin is investigated. This is a simple problem with an available algebraic solution until the flow reaches the mold walls.

Darcy's Law is used for the modeling of the resin flow advancement inside the mold. The media is said to be homogeneous, thus constant porosity is applied for the entire computational domain. The process is also considered isothermal.

The mathematical formulation of the phenomenon described above can be expressed by the Darcy's equation (Equation 1) which correlates the pressure gradient and the velocity within a porous media.

$$
\vec{V}=\frac{\overline{\bar{K}}}{\mu} \nabla P
$$

where $\vec{V}$ is the resin velocity vector $[\mathrm{m} / \mathrm{s}], P$ is the pressure $[\mathrm{Pa}], \overline{\bar{K}}$ is the permeability tensor $\left[\mathrm{m}^{2}\right]$ and $\mu$ is the viscosity [Pa.s].

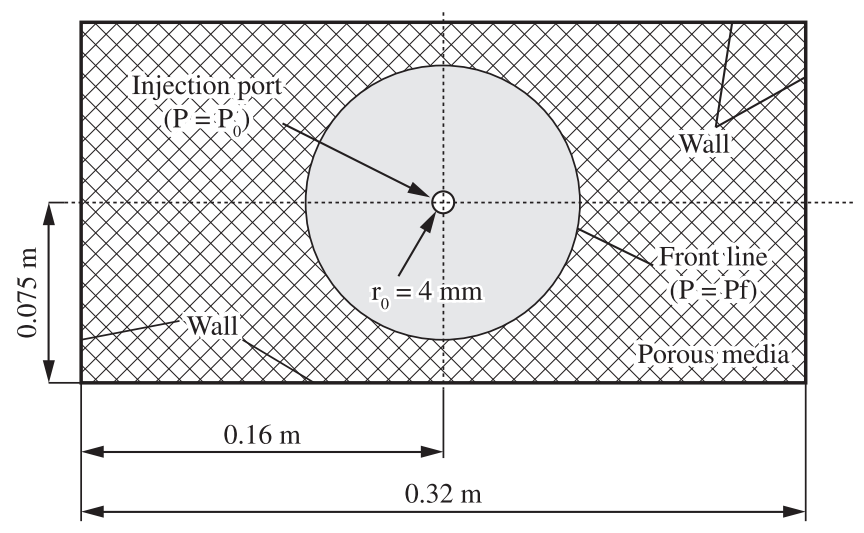

Figure 1. Computational domain for a simple radial problem. 
Typical polymeric resins used in the RTM process show a non-Newtonian behavior. However, for the purpose of the present work (investigation of the progress of the resin front line), a Newtonian approach is completely suitable. Indeed, this consideration is largely used in numerical investigations of the RTM process ${ }^{3,7,26}$, being generally acceptable due to the relatively low velocity of the fluid, the wide channels in which the fluid flows and the low range of shear variation during flow. Thus, assuming the resin as a Newtonian incompressible fluid, the mass conservation equation assumes the form

$$
\nabla \cdot \vec{V}=0
$$

Combining Equations 1 and 2, the equation for the pressure can be written as

$$
\nabla \cdot\left(\frac{\bar{K}}{\mu} \nabla P\right)=0
$$

In Equation 3, media permeability and resin viscosity are kept inside the derivative, which is the most generalist case. In the present solution, resin viscosity is considered constant whereas permeability is considered either isotropic ( $K$ is constant) or orthotropic $\left(K_{x x} \neq K_{y y}\right.$ and $K_{x y}=K_{y x}=0$ ). Equation 3 is numerically solved using the method described in section 2.4

The boundary conditions to be used with Equation 3, also shown in Figure 1, are given by:

- $P=P_{0}$ at the injection point;

- $\partial P / \partial n=0$ at the mold walls ( $n$ is the direction normal to the wall); and

- $P=P_{f}$ at the resin front line, where $P_{f}$ is the front line preset pressure.

A finite volume method ${ }^{10,12,27}$ was used for the solution of Equation 3 inside the gray region of Figure 1, and determine the pressure field gradient throughout the resin impregnated area. The pressure field is then used (in Equation 1) to determine the velocity field and, consequently, the volumetric flow-rate through the control volume surfaces.

\subsection{Transient solution}

The numerical solution of Equation 3 is quite simple, but it must be continuously solved during the simulation. Since Equation 3 does not include a transient term, the transient solution is obtained by successively solving Equation 3 until mold filling is complete.

In this work, the main goal of the simulation is to determine the resin flow-front position as a function of the injection time. The used methodology is very similar to that of well-known finite element/control volume (FE-CV) methods, except that the finite element method is substituted here for a Control Volume Finite Element method (CVFE). The finite volume method already has in its basic formulation the definition of elements and control volumes, each of them composed of four nodes (points), and the control volumes are created around the nodes with the contribution of four different elements. The mass (i.e. resin) crossing each volume is accounted for by integrating the continuity equation through the volume boundaries (surfaces). The discretized domain and the approach followed to create the volumes are shown in Figure 2. The gray area in this figure represents the region already impregnated with the resin.

Monitoring of the advancement of the resin flow-front was obtained using a Flow Analysis Network (FAN) technique ${ }^{17}$. Starting from a condition in which the inlet locations and injection pressure are known, and assuming the pressure for all volumes outside the gray area in Figure 2 equal to zero, solution of Equation 3 will determine the pressure field all over the computational domain. The velocity field is obtained by substituting the pressure field in Equation 1. The flow rates crossing the boundaries of the control volumes can be calculated by multiplying the normal velocity by their respective areas. After that, the smallest time step needed to fill at least one volume, $(\Delta t)_{\min }$, is calculated by

$$
(\Delta t)_{\min }=\min \left(\frac{\forall_{i}-\forall_{i}^{f}(t)}{\dot{\forall}(t)}\right)
$$

where $\forall_{i}$ is the total volume of the finite volume $i, \forall_{i}^{f}(t)=f_{i}(t) \forall_{i}$ denotes the total volume of fluid that has entered volume $i$ at time $t$ and $\dot{\forall}$ is the volumetric flow-rate into volume $i$.

The $(\Delta t)_{\min }$ is then used to determine the filling volume fraction and then a filling factor, $f$, for the control volume $i$ defined as

$$
f_{i}(t+\Delta t)=\frac{\forall_{i}^{f}(t)-\Delta t_{\min } \dot{\forall}(t)}{\forall_{i}}
$$

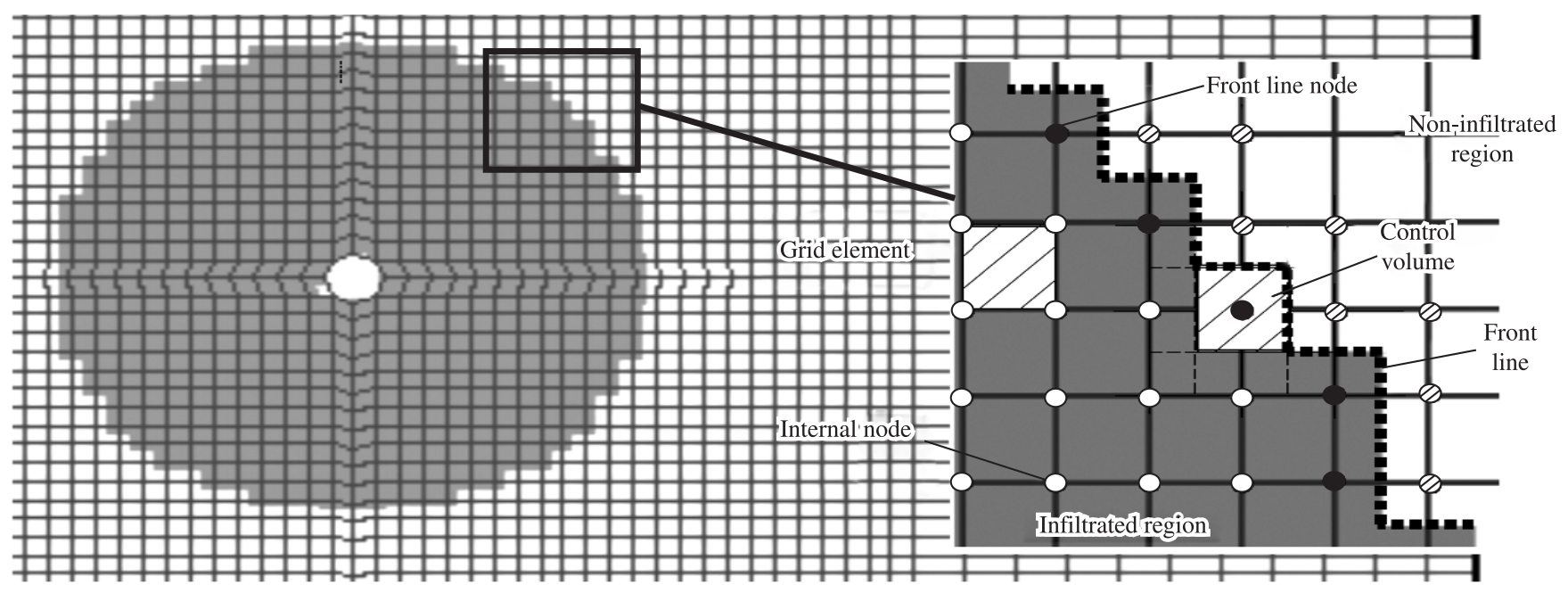

Figure 2. Discretized domain. 
In Figure 2, all volumes with filling factor $f=1$ are painted in gray. It may be noted that the volume boundaries are not plotted and that the grid lines represent the element boundaries (not the volumes). The description of nodes and volumes is shown in the zoomed in region of Figure 2. In this figure, the black nodes indicate the volumes near the resin front line, whereas the white nodes indicate the volumes in the fully resin infiltrated region, which are not in contact with the resin front line. The volumes with hatched nodes have a filing factor defined as $0<f<1$, and therefore are said to be in the non-infiltrated region. The front line position is tracked after each time-step integration, being defined at a position between the last volume inside the resin-infiltrated region (black nodes) and the volumes in the non-infiltrated region (hatched nodes).

For all non-infiltrated volumes, the transport equation (Equation 3) is substituted by

$$
P=P_{f}=0
$$

and the pressure field is determined only for the infiltrated region.

Moreover, solution of the flow front advance algorithm can be summarized as:

1) specify $P=P_{0}$ to all inlet locations;

2) specify $P=P_{f}$ to all empty volumes;

3) solve Equation 3 to obtain the pressure field;

4) calculate the velocity field with Equation 1;

5) determine $\forall_{i}, \forall_{i}^{f}(t), \forall$ and $\Delta t_{\text {min }}$ for all flow front volumes;

6) calculate $f$ for all flow front volumes;

7) if the mold is not completely filled, return to step 2.

\subsection{Front line geometry reconstruction}

Determination of the front line position is sketched in Figure 3. A simple, but precise algorithm was written to interpolate the position of the resin front line inside the partially filled volumes.

For the radial flow problem shown in Figure 1, the exact (algebraic) coordinates of the flow front $\left(x_{a l g}, y_{a l g}\right)$ were plotted inside the grid domain sketched in Figure 3. In this figure, the hatched volume has $f=1$, while the volume with dashed boundaries has $0<f<1$. Based on these coordinates at different volumes and times, Equation 7, proposed in this study, can be used to calculate the numerical coordinates $\left(x_{f}, y_{f}\right)$ inside each partially filled volume of the flow front.

$$
\begin{aligned}
& x_{f}=x+\frac{u}{|u|}(f-0.5) d \\
& y_{f}=y+\frac{v}{|v|}(f-0.5) d
\end{aligned}
$$

As shown in Equation 7, the coordinates of the front line position $\left(x, y_{f}\right)$ are obtained as a function of the volume center coordinates $(x, y)$, the velocities at this point, the filling factor $f$ and a scale parameter $2 d=\sqrt{2 A}$, where $A$ is the area of the 2D volume and $d$ is the diagonal of a square volume.

Using Equation 7 for volume $j$ in Figure 3, which is partially filled, i.e. with $0<f<1$, the resin front line position $\left(x_{f}, y_{f}\right)$ will pass through a point between the center of volumes $i$ and $j$. Moreover, if $0.5<f<1$, which means that more than half of the volume $j$ is filled with resin, Equation 7 will calculate $\left(x, y_{f}\right)$ in a position further from the center of volume $j$. The other three possibilities are:

- If $f=0,\left(x_{f} y_{f}\right)$ will be a point halfway between volumes $i$ and $j$;

- If $f=1,\left(x_{f}, y_{f}\right)$ will be close to the center of element $j$;

- If $f=0.5,\left(x_{f}, y_{f}\right)$ will be the exact coordinates of the center of volume $j(x, y)$.
This simple strategy may be easily implemented, being less time-consuming than more refined geometry reconstruction methods.

\subsection{Mass conservation across the grid volumes}

Mass conservation across grid volumes is probably the most important and difficult parameter to be controlled during simulation. In general, Equation 3 can be easily solved with any finite element or finite volume method and good results may be obtained even for coarse grids. The problem with mass conservation lies on the difficulty of determining the flow through the volume surfaces. Assuming that Equation 1 provides a good approximation for the velocity field, the flow through the volume boundaries could, in theory, be calculated with sufficient accuracy.

The most common approach is to create control volumes centered at the grid nodes. In this work, the control volumes were built with the contribution of four different elements in such a way that their boundaries are in the middle of these elements. Thus, a local system of coordinates ( $s$ and $t$ in Figure 4 ) is used to calculate the pressure derivatives needed to solve Equation 1.

Inside the fully filled volumes $(f=1)$, mass conservation is obtained with the finite volume formulation. In this method, the mass flow is integrated over the whole volume and mass conservation is enforced for all grid volumes. The term inside the brackets in Equation 3, if multiplied by the density, becomes

$$
\rho \frac{\bar{K}}{\mu} \nabla P \rightarrow\left[\frac{k g}{s} \frac{1}{m^{2}}\right]
$$

If this property balance (inlet equal to outlet for a steady flow) is guaranteed, the resulting pressure field will approximate mass conservation through the control volumes. As described in section 2.4, Equation 3 is integrated over the control volume and the resulting volume integral is converted into a surface integral that will be evaluated over the control volume faces.

For the volumes in the front line $(0<f<1)$, it is necessary to determine in which faces the resin is entering or leaving this control volume. For the flow characteristics shown in Figure 5, it is not possible

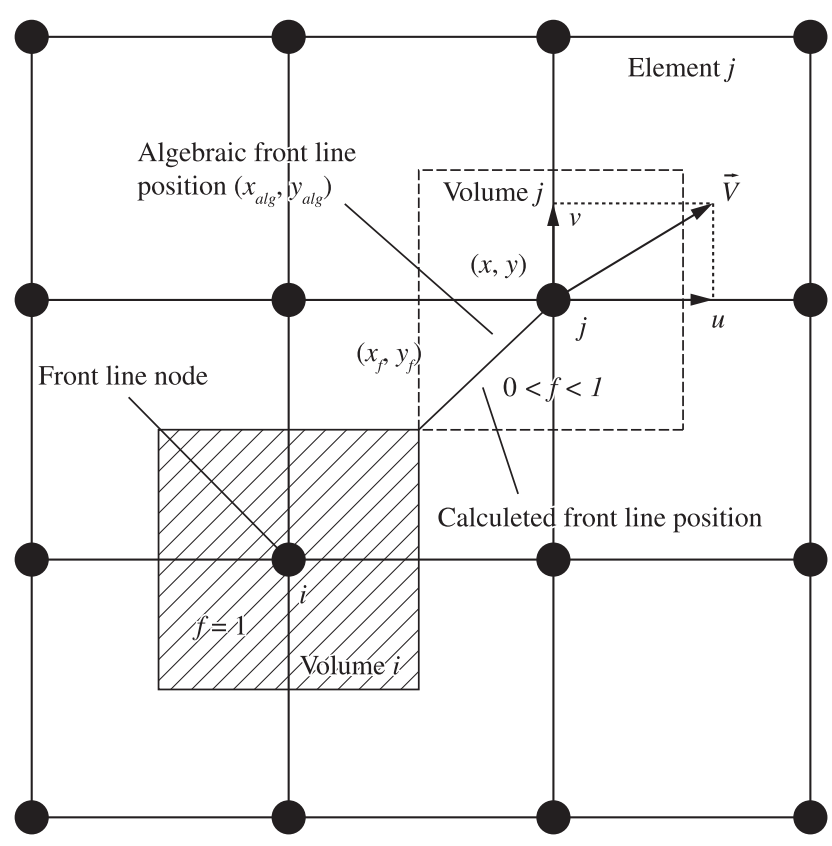

Figure 3. Front line determination. 
to know in advance the exact position of the flow front inside the volume $i$, therefore the vectors drawn in Figure 5 show resin entering by the West face and leaving through the North face of the volume. Faces South and East are said to be in a domain region without resin. Note that velocity is only defined in the region partially or fully filled with resin. The calculated pressure gradients in faces South and East of this volume are not null, and Equation 1 will determine a non-zero velocity vector for these faces. To avoid the calculation of mass flux through these faces, a criteria must be established to determine which fluxes will be considered in the mass balance of volume $i$. A reasonable and precise criteria is to consider that flow flux may only occur through the faces that belong to at least one volume with $f=1$. This criteria will not

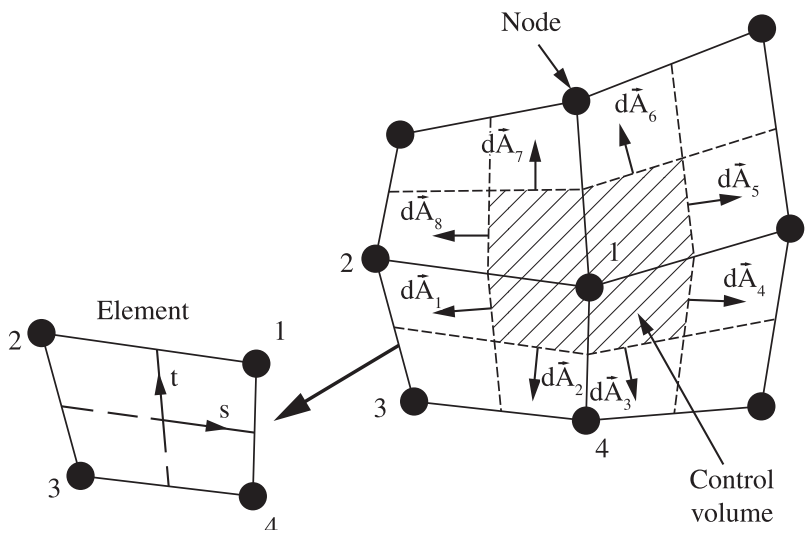

Figure 4. Control volume formation. verify for all volumes in the grid, but the errors involved in the mass flow calculations are expected to be very small. As shown later, the results of this work validate this approximation. The procedure followed for the calculation of mass fluxes through the control volumes is also simple and may be easily implemented.

\subsection{Numerical solution of the pressure equation}

In the present formulation, a finite volume method that includes finite elements in its formulation was used to solve the pressure equation ${ }^{10-12,27}$. The computational domain was discretized with four-node rectangular elements. For each node, a control volume is created with the contribution of four different elements, as shown in Figure 4. A local coordinate system $(s, t)$ is created inside each element and all variables and derivatives inside the element can be expressed using this system.

The most fundamental concept of any finite volume method lies on the integration of all differential equations over the discretized control volumes. Thus, for each control volume of the grid, Equation 3 becomes

$$
\int_{V \cdot C} \nabla \cdot\left(\frac{\bar{K}}{\mu} \nabla P\right)=0
$$

The divergence theorem states that the above volume integral can be replaced by a surface integral such as

$$
\int_{S}\left(\frac{\overline{\bar{K}}}{\mu} \nabla P\right) \cdot d \vec{A}=0
$$

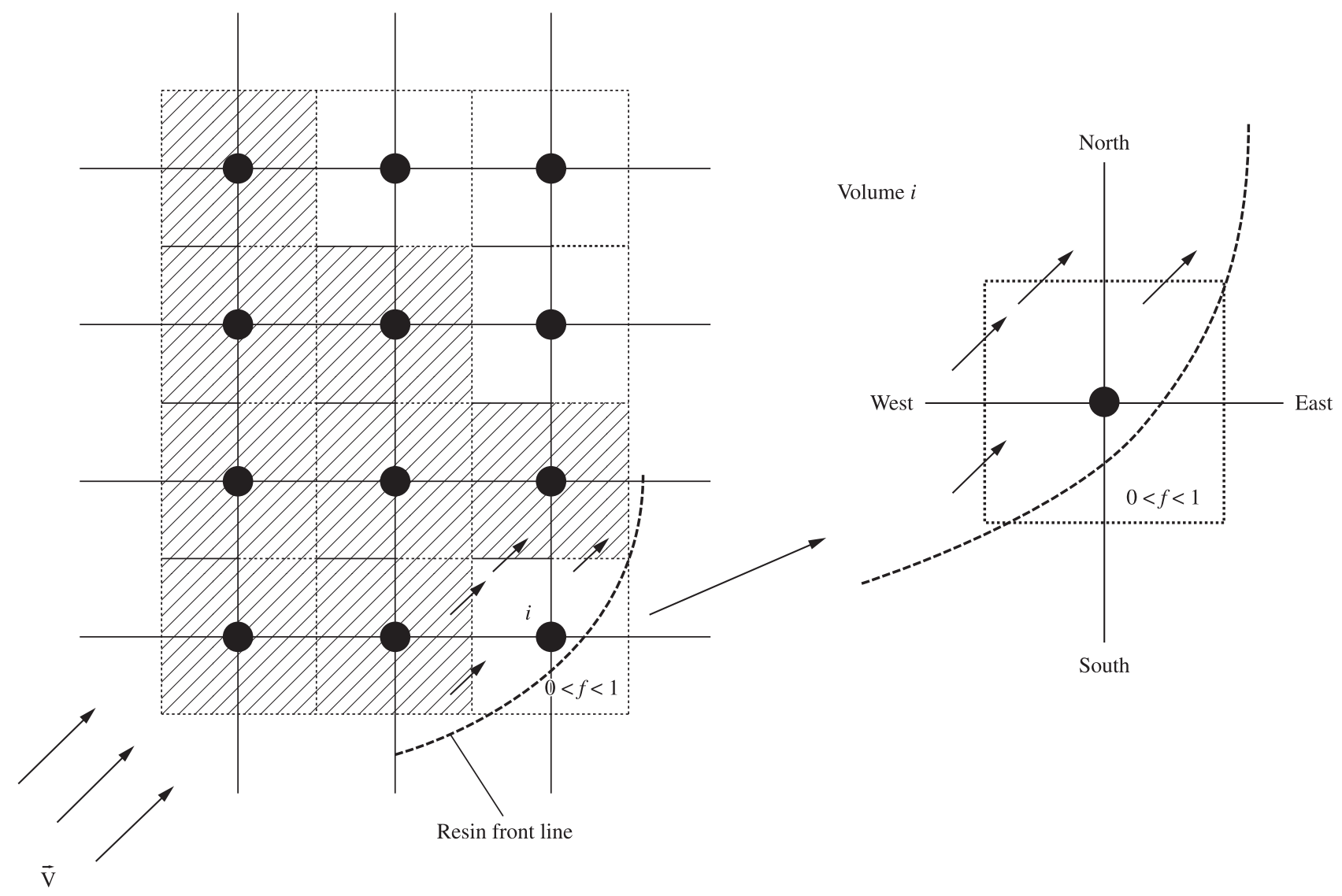

Figure 5. Mass flow through the control volumes. 
Integration of Equation 10 over the control volume shown in Figure 4 results in

$$
\int_{S}\left(\frac{\overline{\bar{K}}}{\mu} \nabla P\right) \cdot d \vec{A}=\sum_{i=1}^{8} \int_{S_{i}}\left(\frac{\overline{\bar{K}}}{\mu} \nabla P\right) \cdot d \vec{A}_{i}=0
$$

where the index $i$ represents each one of the eight faces of the control volume shown in Figure 4.

Each integral in the right side of Equation 11 can be expressed using a generic matrix notation as

$$
\int_{S_{i}}\left(\frac{\overline{\bar{K}}}{\mu} \nabla P\right) \cdot d \vec{A}_{i}=\frac{1}{\mu}\left[\begin{array}{ll}
K_{x x} & K_{x y} \\
K_{y x} & K_{y y}
\end{array}\right]\left[\begin{array}{l}
\partial P / \partial x \\
\partial P / \partial y
\end{array}\right] \cdot\left[\begin{array}{l}
\Delta y \\
-\Delta x
\end{array}\right]=0
$$

Finally, for each volume face

$$
\begin{aligned}
& \int_{S_{i}}\left(\frac{\overline{\bar{K}}}{\mu} \nabla P\right) \cdot d \vec{A}_{i}=\frac{1}{\mu}\left(K_{x x} \frac{\partial P}{\partial x}+K_{x y} \frac{\partial P}{\partial y}\right) \Delta x- \\
& -\frac{1}{\mu}\left(K_{y x} \frac{\partial P}{\partial x}+K_{y y} \frac{\partial P}{\partial y}\right) \Delta y=0
\end{aligned}
$$

Shape functions are defined inside each element based on the local coordinate system. The pressure at any position $(s, t)$ inside the element can be approximated as a function of the nodal calculated variables ${ }^{10}$ as

$$
P_{(s, t)}=\frac{1}{4}\left[P_{1} N_{1}+P_{2} N_{2}+P_{3} N_{3}+P_{4} N_{4}\right]
$$

where $N_{1}=0.25(1+\mathrm{s})(1+t), N_{2}=0.25(1-s)(1+t), N_{3}=0.25(1-s)$ $(1-t)$ and $N_{4}=0.25(1+s)(1-t)$.

The pressure derivatives are also defined as a function of the local coordinates by

$$
\left.\frac{\partial P}{\partial x}\right|_{(s, t)}=\sum_{i=1}^{4} \mid \frac{\partial N_{i}}{\partial x} \quad P_{(s, t)}
$$

More detailed information about the shape function can be found in the literature for finite elements $\mathrm{s}^{28,29}$ or finite volumes ${ }^{10,27}$.

The combination of Equations 13, 14 and 15 results in a linear system of equations. The resulting coefficient matrix is sparse, but the solution has proven to be stable and fast. An ILU (Incomplete LU) decomposition method ${ }^{30}$ was used to solve this linear system.

\section{Results}

The numerical methodology described above was validated by solving three case studies. The first one is the radial injection problem, with an algebraic solution. The second and the third cases do not have an algebraic solution, therefore the results are compared with those of a commercial general purpose CFD (computational fluid dynamic) package - FLUENT, in order to evaluate the ability of the present methodology in solving more complex problems.

The general parameters used in all simulations are presented in Table 1. The injected fluid (resin) is considered Newtonian, with constant viscosity $(\mu)$ and density $(\rho)$. Two different conditions are set for the porous media: isotropic $\left(K_{x x}=K_{y y}\right)$, cases 1 and 2, and orthotropic $\left(K_{x x} \neq K_{y y}\right)$, case 3 .

\subsection{Radial infiltration (Case 1)}

Figure 1 shows a sketch of the radial infiltration problem. The injection point is located close to the geometrical center of the mold. For this simple case, the numerical results can be compared with the analytical solution $^{31}$ (until the resin reaches the mold walls), which is given by

$$
t=\frac{\mu}{2 K P_{0}}\left[r^{2} \ln \left(\frac{r}{r_{0}}\right)-\frac{1}{2}\left(r^{2}-r_{0}^{2}\right)\right]
$$

where $P_{0}$ is the pressure at the injection point [Pa], $t$ is the time [s], $r$ the position (radius) of resin front line [m] and $r_{0}$ the radius of the injection port $[\mathrm{m}]$.

The analytical and numerical solutions are compared in Figure 6. Both solutions are nearly identical in the first few seconds of the simulation (Figure 6a), later distancing from each other due to errors in the mass flow calculation along the control volumes surfaces. Since the numerical solution consists of a time integration procedure (not iterative), all errors propagate until the end of the simulation. As expected, this effect can be minimized by using more refined grids.

To quantify the deviation between solutions, a second figure is presented (Figure 6b), showing the relative error between the numerical $\left(x_{\text {num }}\right)$ and algebraic $\left(x_{\text {alg }}\right)$ solutions, as defined in Equation 17. This error is quite significant for the coarser grid, becoming considerably smaller for the more refined grids.

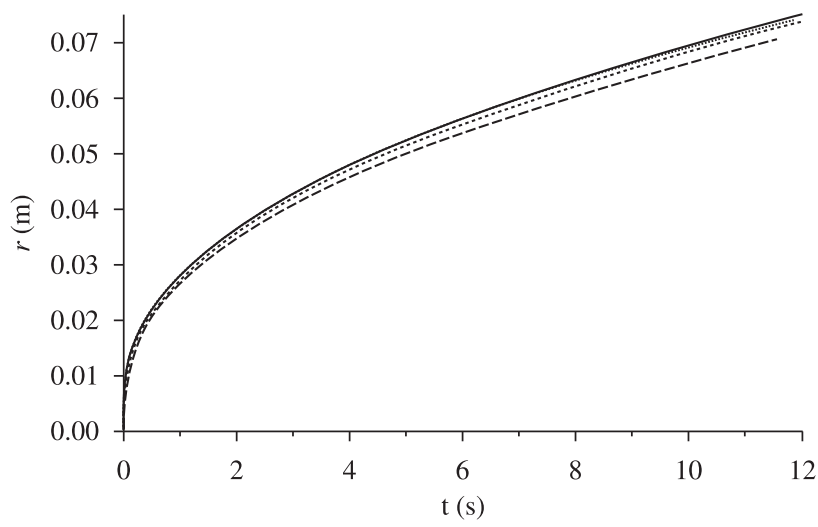

(a)
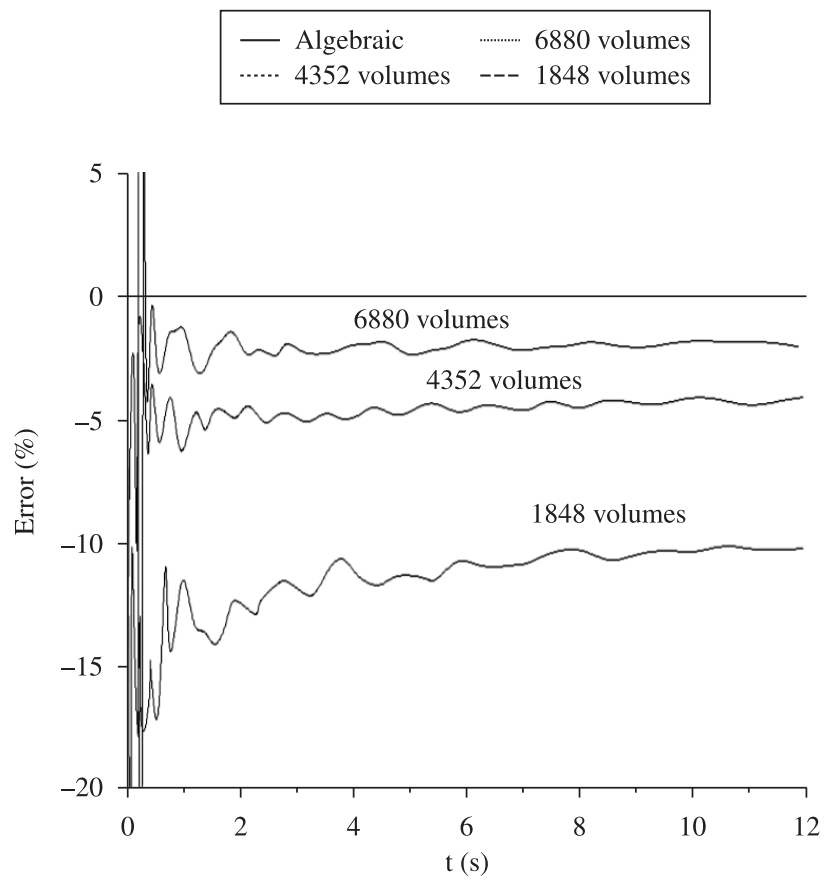

(b)

Figure 6. Radial flow solution: a) front line position; and b) relative error between analytical and numerical solutions. 


$$
\operatorname{Error}(\%)=100 \frac{\left|x_{\text {num }}-x_{\text {alg }}\right|}{x_{\text {num }}}
$$

To analyze the resin front line position and also the errors in the mass flow calculations, a zoomed region, close to the resin front line, is presented in Figure 7. The gray area again represents the domain region fully filled with resin, i.e. for $f=1$ (actually, $f>0.999$ ). Next to that region, there is a borderline zone where all volumes show $0<f<1$. Some of these volumes are highlighted (with dashed lines) and the respective $f$ values are shown close to each respective upper right corner. The calculated front line position (Equation 7) inside each volume is indicated in Figure 7 with the "+" symbol. The position of the numerical front line (r), plotted in Figure 6a can be visualized in Figure 7, which also shows the algebraic position of the resin front line for comparison.

Analysis of the control volume called $1610(f=0.076)$, shows that resin is considered to be entering this volume by 4 out of 8 boundary faces. It is also assumed that no resin is leaving this volume through its faces. The volume 1610 is almost empty, and therefore the mass flow that could eventually leave volume 1610 and enter volume 1611 or 1629 may be disregarded in the mass flow balance of volume 1610 .

Table 1. General simulation conditions.

\begin{tabular}{cc}
\hline Property/Variable & Value \\
\hline$r_{0}$ & $0.004 \mathrm{~m}$ \\
$P_{0}$ & $1 \mathrm{bar}$ \\
$K_{x x}$ & $3 \times 10^{-10} \mathrm{~m}^{2}$ \\
$K_{y y}$ & $3 \times 10^{-11} \mathrm{~m}^{2}$ \\
$K_{x y}=K_{y x}$ & $0 \mathrm{~m}^{2}$ \\
$\mu$ & $0.07 \mathrm{~Pa} \mathrm{~s}$ \\
$\varepsilon$ & 0.75 \\
$\rho$ & $920 \mathrm{~kg} \cdot \mathrm{m}^{3}$ \\
\hline
\end{tabular}

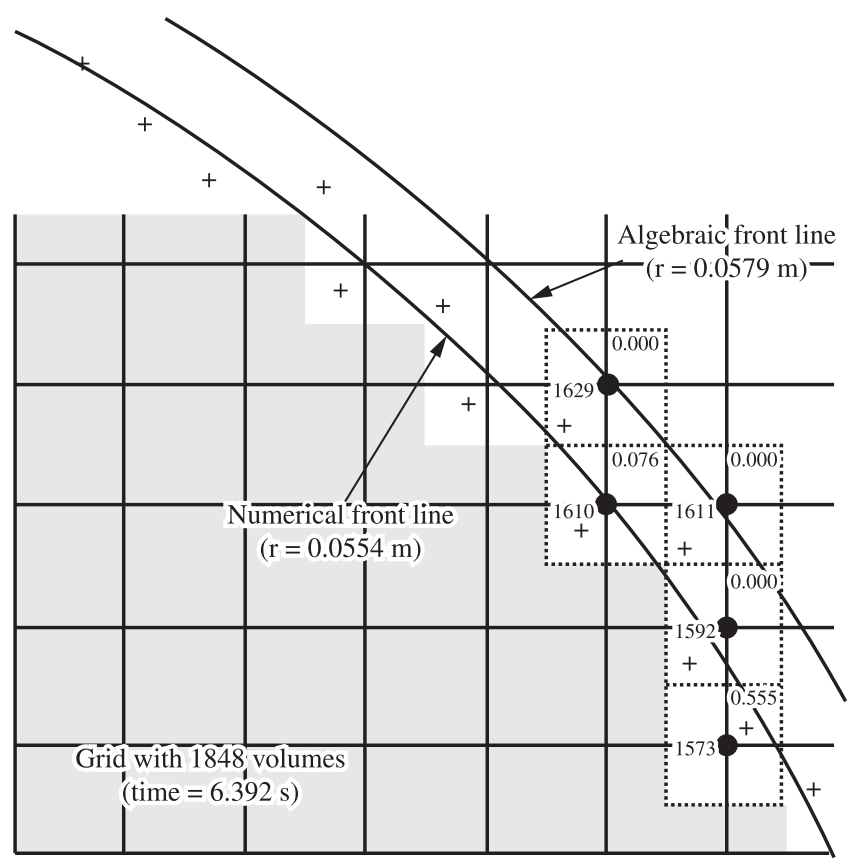

Figure 7. Determination of the resin front line position.
According to Figure 7, volume 1611 will not show any flow entering or leaving its boundaries at that time step and, as a consequence, will remain empty on the following time step. This is exactly what happened with volume 1592 one time step earlier to that.

The above paragraph describes the approximations in the mass flow calculation of the proposed method. As shown in Figure 6, coarse grids yield larger errors that diminish following grid refinement. However, good results may be obtained even with a coarse grid. The proposed methodology has already been compared with the VOF method ${ }^{32}$ and the results (Figure 8) showed that the number of volumes required by the former method to achieve the desired accuracy is considerably smaller. Note that in Figure 8, the coarser grid has 5328 volumes which nearly represent the more refined grid in Figure 6, preventing visualization of the relative error between the numerical and algebraic solutions.

\subsection{Two-dimensional mold infiltration in an isotropic media (Case 2)}

The computational domain shown in Figure 9 represents another actual lab-scale mold geometry. In this solution, the injection hole is near the left wall and there are no pre-set output ports. The simulation is interrupted when all grid volumes are fully filled. From the circular injection port, a radial flow pattern can be observed in the very beginning of the simulation. When the resin reaches the top and bottom walls, the front line becomes progressively closer to a straight line, normal to these walls, and a rectilinear (1-D) advancement of the flow front is then observed. After that, the $\mathrm{x}$-coordinate of the front line position $\left(x_{f}\right)$ can be determined as a function of the injection time $(\mathrm{t})$.

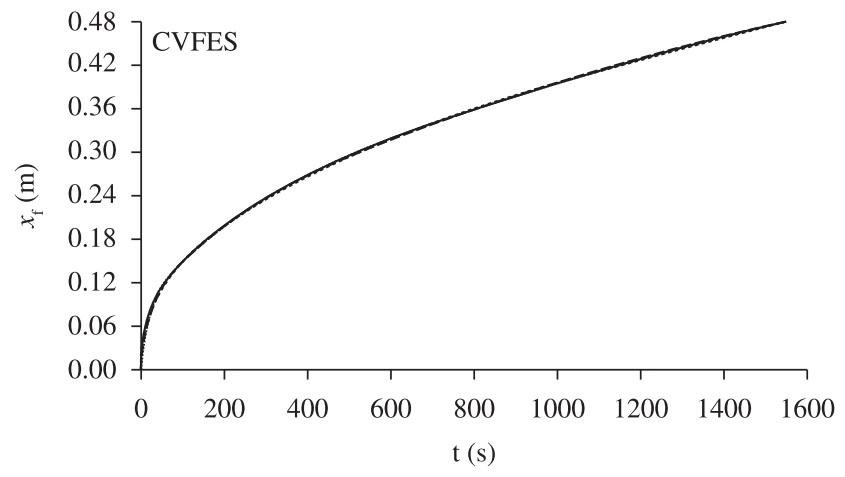

(a)

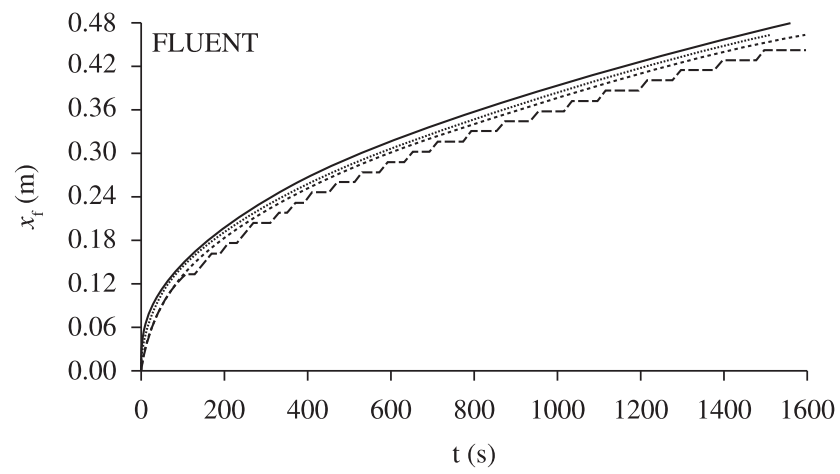

(b)

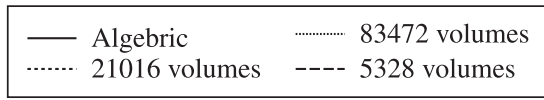

Figure 8. Radial flow solution for the CVFES and FLUENT applications ${ }^{32}$. 
Even for this simple geometry, the region close to the injection port does not have an analytical solution. Results obtained with the developed code, which will hereafter be called CVFES (Control Volume Finite Element Solver), were compared with the solution obtained with the FLUENT software ${ }^{23}$. In this software, the VOF method $^{26,33}$, combined with the porous media formulation, is used to track the advancement of the resin inside the mold cavity. Identical grids were used in both codes. In addition, it is important to bear in mind that the use of the FLUENT software for RTM problems has already been validated ${ }^{32}$.

The position of the resin front along the horizontal symmetry line shown in Figure 9 as a function of time is used to compare the results obtained with the two codes. Two different grids were used in the simulations: i) all elements with the same size (Figure 10a), and ii) a more refined grid in the region close to the injection port, $x<0.1$ $\mathrm{m}$ (Figure 10b). In this region, the elements are three times smaller than in the $0.10 \mathrm{~m}<x<0.32 \mathrm{~m}$ region. Grid refinement was carried out for both meshes. Simulations were performed using the mesh of Figure 10a with 5340 and 21984 elements. For the configuration shown in Figure 10b, grids with 2498, 4026, 5916 and 8534 elements were evaluated.

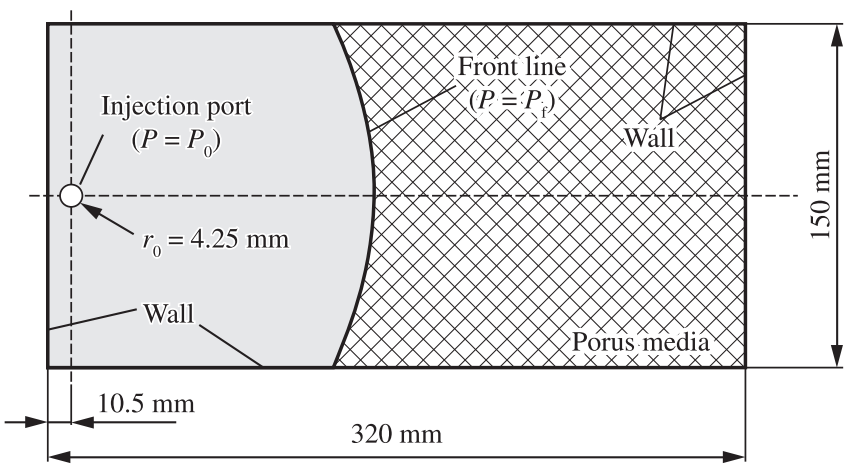

Figure 9. Computational domain for a 2D mold injection.

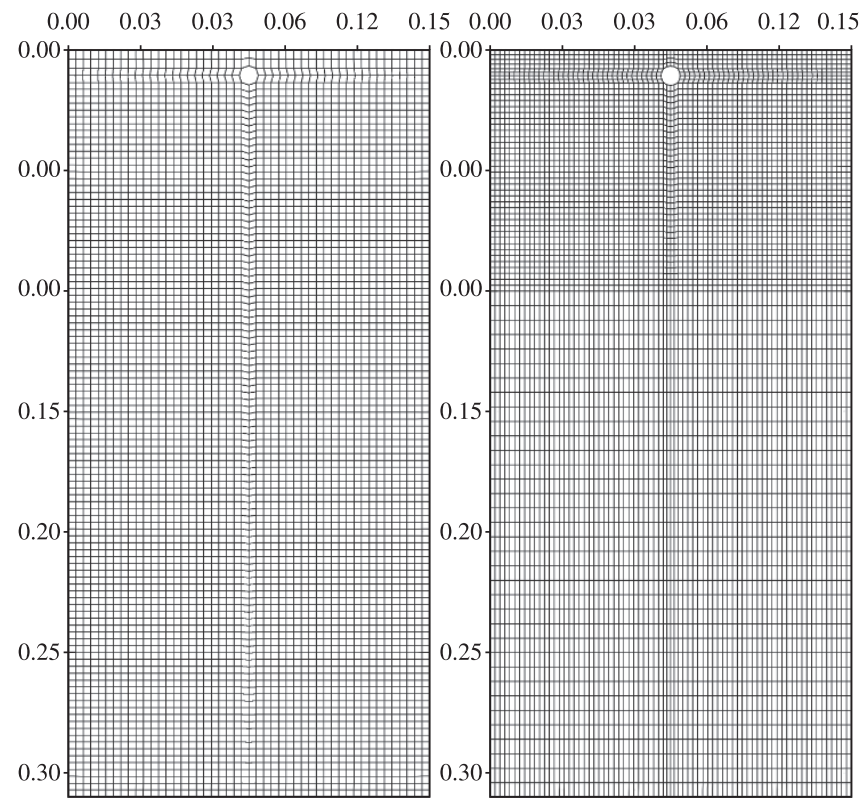

Figure 10. Grid used with the 2D mold injection problem: a) Regular grid; and b) Refined grid near the injection port.
Initially, the grid shown in Figure 10b was chosen for the simulations. Grid refinement in the regions with larger gradients in the variables fields (pressure, in this case) is a common practice when solving CFD problems. This technique helps reducing the number of grid elements and, consequently, simulation time.

The solutions obtained with the grid presented in Figure 10b showed a particular behavior. Both solutions were very similar for the first 15 seconds of the simulation. However, the flow advancement calculated with FLUENT (VOF) seems to slow down at the boundary of the region of more refined grid (at $x_{f}=0.1 \mathrm{~m}$ ), whereas the CVFES solution does not display this behavior in any of the tested grids.

Figure $11 \mathrm{~b}$ displays the results for a refined grid with 8534 elements. In this figure, it is possible to observe that while the CVFES solution remains locally unchanged (the flow front advance is linear with time), the VOF solution obtained with FLUENT changes abruptly at $x_{f}=0.1 \mathrm{~m}$ and then returns to its previous behavior, with a trend similar to that of the CVFES solution. This was noticed even though a grid refinement test had been previously performed and the solution was found to be grid-independent for meshes with more than 5916 volumes.

The regular mesh shown in Figure 10a was used to evaluate the grid refinement effect on the solution and the results are plotted in Figure 11a. Analysis of this figure leads to the conclusion that the CVFES solution seems unaffected by grid size change, whereas the solution obtained with the VOF method, from FLUENT, showed to

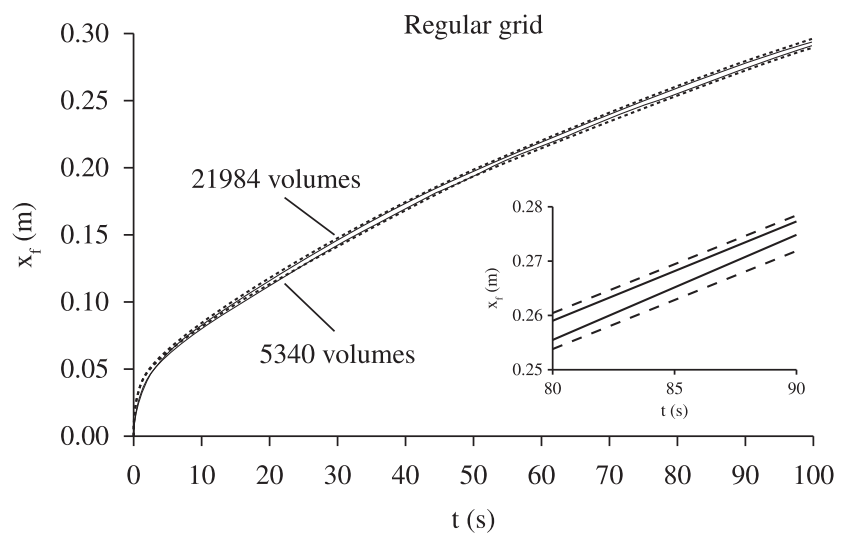

(a)

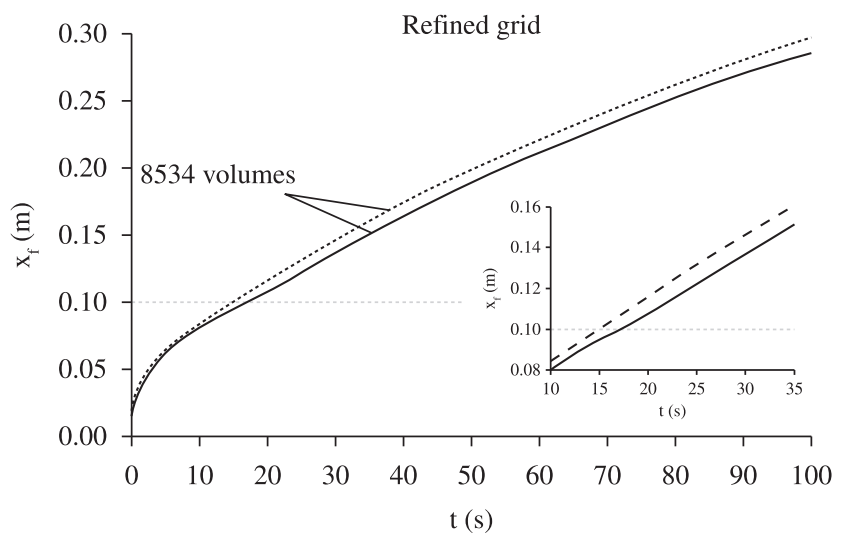

(b)

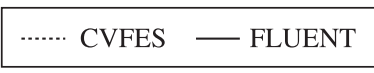

Figure 11. Front line position along the symmetry line shown in Figure 9 (isotropic): a) regular grid; and b) refined grid. 


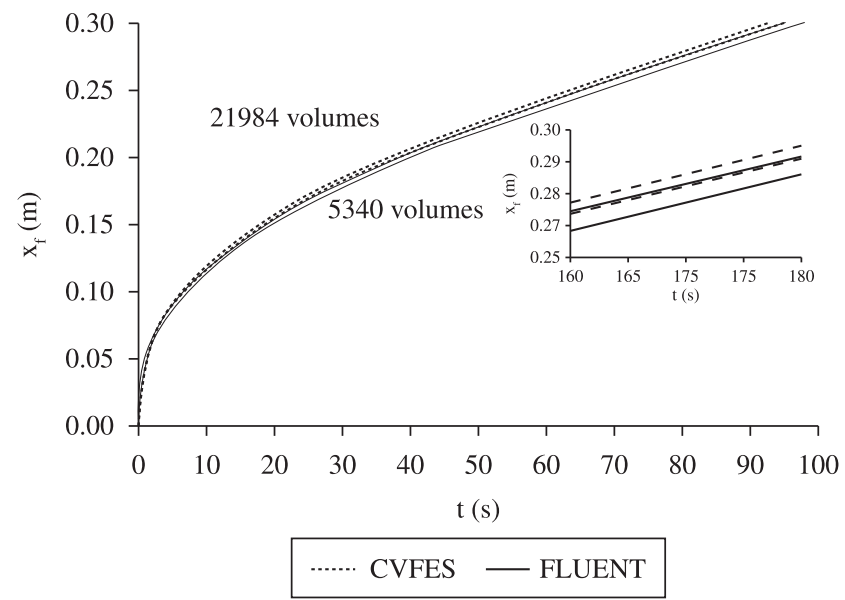

Figure 12. Comparison between the orthotropic and isotropic solutions.

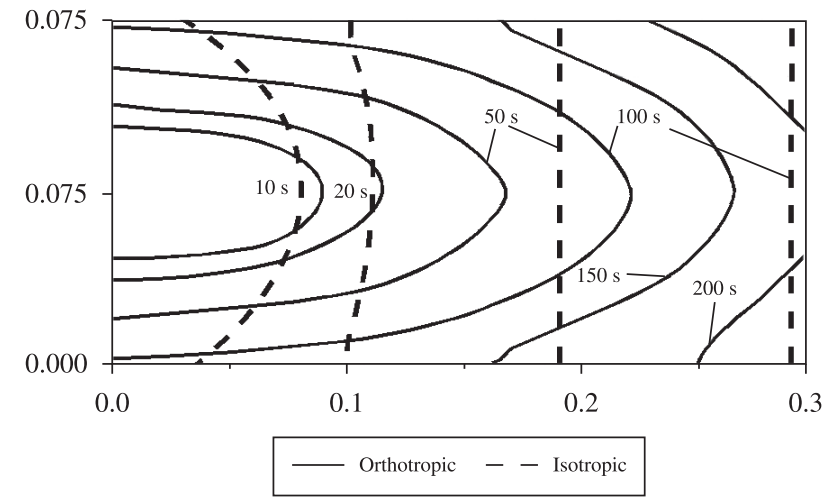

Figure 13. Front line position along the symmetry line shown in Figure 9 (orthotropic).

be dependent. Since a smooth element size change is generally used, a pronounced effect is not usually observed.

A deeper investigation of this phenomenon is out of the scope of this work. Nevertheless, this observation helps highlighting the benefits of the solution obtained with the present formulation, i.e. in the CVFES solution, resin mass conservation through the faces of the volumes is expected even within regions with large variations in grid volume size. In addition, Figure 11a shows almost identical results for both methods and, therefore, the solution obtained with the CVFES is also considered validated for this problem.

\subsection{Two-dimensional mold infiltration in an orthotropic media (Case 3)}

This test problem uses the same geometry presented in Figure 9 and is nearly the same problem except that the permeability in the $x$ direction $\left(K_{\mathrm{xx}}\right)$ now differs from the permeability in the $y$ direction $\left(K_{\mathrm{yy}}\right)$ (see Table 1). This kind of behavior is found when in-plane unbalanced layers are used ${ }^{2,34}$ or when different reinforcements are stacked together along the thickness, making up a so-called laminate (in this case $K_{\mathrm{zz}}$ is also important).

In this study, a single reinforcement layer is considered to have distinct permeabilities in each coordinate axis. This problem was used to evaluate the computational implementation of a non-isotropic permeability tensor and the results were again compared with the solution obtained with FLUENT for the grid shown in Figure 10a. The front line position $\left(x_{f}\right)$ is plotted along the horizontal symmetry line (Figure 12 at $y=0.075 \mathrm{~m}$ ) for the grids with 5340 and 21984 volumes.

The permeability in the $y$ direction was preset ten times lower than in the $x$ direction, i.e. resin will flow faster in the $x$ direction than in the $y$ direction. In addition, the overall resistance to resin flow is greater than in the isotropic case and, because of that, the resin takes longer to reach the right wall of the mold. Even though the geometry in Cases 2 and 3 are identical, the orthotropic solution is more complex than the isotropic one. The results in Figure 13 show a reasonable quantitative agreement between the two numerical solutions $\left(x_{f}^{F L U E N T}-x_{f}^{C V F E S}<1 \mathrm{~cm}\right)$.

The resin front line position at different times for the isotropic and orthotropic problems are illustrated in Figure 13. For the isotropic problem, the resin quickly reaches the top and bottom mold wall and its front line becomes a straight line normal to these walls, whereas this does not occur for the orthotropic problem, yielding an elliptic shape flow pattern.

\section{Conclusions}

This work presents an alternative methodology for the tracking of the resin flow-front within a mold cavity applied to the resin transfer molding of composite materials. The proposed methodology uses a control volume finite element method to determine the pressure gradient inside the porous (fibrous) media and the flow analysis network (FAN) technique to determine the filling of the volumes next to the resin front line. This methodology focuses on how to evaluate the resin mass flow through the faces of the volumes and how to reconstruct the flow front geometry.

The results showed that using a finite volume method to evaluate the pressure gradients inside the mold ensures mass conservation through the internal (fully filled) volumes. Thus, the mass balance inconsistency is nearly restricted to the flow front volumes. For the volumes in the front line $(0<\mathrm{f}<1)$, an algorithm was proposed to determine in which faces the resin would enter or leave the control volume, improving the evaluation of the mass flow through its faces and also reducing the mass imbalance of the flow front volumes.

A detailed description of the method is reported along with three validation problems, the first had an analytical solution and the others were solved with the aid of the FLUENT software for comparison. All of them showed a very good qualitative and quantitative agreement. In all, the proposed methodology combines simple formulation and easy computational implementation, and the obtained results showed a good estimate of the mass flow across the faces of the volumes, ensuring mass conservation even for coarse grids and for abrupt variations in the size of the grid elements.

\section{Acknowledgments}

The authors are thankful to CAPES, CNPq and FAPERGS for the financial support.

\section{References}

1. Souza JA, Rocha LAO and Amico SC. Numerical Simulation of the Resin Transport Through Fiber Reinforcement Medium. In: Proceedings of the 19th International Congress of Mechanical Engineering; 2007; Brasília, Brazil. Rio de Janeiro: ABCM, 2007.

2. Souza JA, Nava MJA, Rocha LAO and Amico SC. Two-Dimensional Control Volume Modeling of the Resin Infiltration of a Porous Medium with a Heterogeneous Permeability Tensor. Materials Research. 2008; 11(3):261-268. http://dx.doi.org/10.1590/S1516-14392008000300006

3. Jinlian H, Yi L and Xueming S. Study of Void Formation in Multi-layer Woven Fabric. Composites: Part A. 2004; 35:595-603. http://dx.doi. org/10.1016/j.compositesa.2003.11.007 
4. Endruweit A and Ermanni P. The in-plane permeability of sheared textiles. Experimental observations and a predictive conversion model. Composites: Part A. 2004; 35:439-451. http://dx.doi.org/10.1016/j. compositesa.2003.11.002

5. Trochu F, Ruiz E, Achim V and Soukane S. Advanced numerical simulation of liquid composite molding for process analysis and optimization. Composites: Part A. 2006; 37:890-902. http://dx.doi. org/10.1016/j.compositesa.2005.06.003

6. Simacek P and Advani SG. A numerical model to predict fiber tow saturation during liquid composite molding. Composites Science and Technology. 2003; 63:1725-1736. http://dx.doi.org/10.1016/S02663538(03)00155-6

7. Shojaei A. Numerical study of filling process through multilayer preforms in resin injection/compression molding. Composites Science and Technology. 2006; 66:1546-1557. http://dx.doi.org/10.1016/j. compscitech.2005.11.035

8. Maliska CR. Transferência de Calor e Mecânica dos Fluidos Computacional. Rio de Janeiro: LTC - Livros técnicos e científicos S. A; 2004.

9. Patankar SV. Numerical Heat Transfer and Fluid Flow. McGraw-Hill Book Company, 1980.

10. Schneider GE and Raw MJ. Control volume finite element procedure for heat transfer and fluid flow using collocated variables: 1. Computational procedure. Numerical Heat Transfer. 1987; 11(4):363-390. http://dx.doi. org/10.1080/10407788708913560

11. Souza JA and Maliska CR. Analysis of a Volume Based Finite Element Methodology in View of the Interpolation Function Employed and Coupling Characteristics. In: Proceedings of the 8th Brazilian Congress of Thermal Sciences and Engineering - ENCIT; 2000; Porto Alegre, Brazil. Porto Alegre: ABCM; 2000. p. 1-11.

12. Ansys. Solver Theory Guide. ANSYS Inc.; 2009. chap. 9.

13. Joshi SC, Lam YC and Liu X. Mass conservation in numerical simulation of resin flow. Composites Part A: Applied Science and Manufacturing. 2000; 31(10):1061-1068. http://dx.doi.org/10.1016/ S1359-835X(00)00067-1

14. Bruschke MV and Advani SG. A numerical approach to model nonisothermal viscous flow through fibrous media with free surfaces. International Journal for Numerical Methods in Fluids. 1994; 19(7):575603. http://dx.doi.org/10.1002/fld.1650190704

15. Joshi SC. Reducing Loss of Resin Flowing in Porous Fibrous Media in Simulation of Composites Fabrication. Polymer Composites. 2010; 31(2):226-235.

16. Versteeg $\mathrm{H}$ and Malalasekra M. An Introduction to Computational Fluid Dynamics: The Finite Volume Method. Prentice Hall; 2007.

17. Phelan Junior, FR. Simulation of the injection process in resin transfer molding Polymer Composites. 1997; 18(4):460-476. http://dx.doi. org/10.1002/pc. 10298

18. Hirt CW and Nichols BD. Volume of fluid (VOF) method for the dynamics of free boundaries. Journal of Computational Physics. 1981; 39(1):201225. http://dx.doi.org/10.1016/0021-9991(81)90145-5

19. Luoma JA and Voller VR. An explicit scheme for tracking the filling front during polymer mold filling. Applied Mathematical Modelling. 2000; 24(8-9):575-590. http://dx.doi.org/10.1016/S0307-904X(00)00004-4
20. Yang J, Jia Y, Ding Y, He H, Shi T and An L. Edge effect in RTM Processes Under Constant Pressure Injection Conditions. Journal of Applied Polymer Science. 2010; 118(2):1014-1019.

21. Li J, Zhang C, Liang R, Wang B and Walsh S. Modeling and analysis of thickness gradient and variations in vacuum-assisted resin transfer molding process. Polymer Composites. 2008; 29(5):473-482. http:// dx.doi.org/10.1002/pc.20439

22. Kim M and Lee W. A new VOF-based numerical scheme for the simulation of fluid flow with free surface. Part I: New free surface-tracking algorithm and its verification. International Journal for Numerical Methods in Fluids. 2003; 42(7):765-790. http://dx.doi.org/10.1002/fld.553

23. Ansys. AnsysFluent 6.3. Available from: <http://www.fluent.com/ software/fluent/index.htm>. Acess in: 23/02/2010.

24. Park J, Kim S, Kim M and Lee W. Finite element analysis of flow and heat transfer with moving free surface using fixed grid system. International Journal of Computational Fluid Dynamics. 2005; 19(3):263-276. http:// dx.doi.org/10.1080/10618560410001730296

25. Zhao H, Ohnaka I and Zhu J. Modeling of mold filling of Al gravity casting and validation with X-ray in-situ observation. Applied Mathematical Modelling. 2008; 32(2):185-194. http://dx.doi. org/10.1016/j.apm.2006.11.009

26. Yang J, Jia YX, Sun S, Ma DJ, Shi TF and An LJ. Enhancements of the simulation method on the edge effect in resin transfer molding processes. Materials Science and Engineering: A. 2008; 478(1-2):384-389. http:// dx.doi.org/10.1016/j.msea.2007.07.044

27. Souza JA. Implementação de um método de volumes finitos com sistema de coordenadas locais para a solução acoplada das equações de NavierStokes. [Tese]. Florianópolis: Universidade Federal de Santa Catarina; 2000.

28. Reddy JN and Gartling DK. The Finite Element Method in Heat Transfer and Fluid Dynamics. Boca Raton: CRC Press; 1994.

29. Zienkiewicz OC and Taylor RL. The Finite Element Method. London: McGraw-Hill; 1989.

30. Netlib Repository at UTK and ORNL. Available from: <http://www. netlib.org/y12m/index.html>. Acess in: 01/2011.

31. Rudd CD. Liquid Moulding Technologies: Resin Transfer Moulding, Structural Reaction Injection Moulding and Related Processing Techniques. Woodhead Publishing. 2005.

32. Silva FMV, Souza JA, Rocha LAO and Amico SC. Comparison of Two Numerical Methodologies for the Modeling of the RTM Process. In: Proceedings of the 12th Brazilian Congress of Thermal Sciences and Engineering - ENCIT; 2008; Belo Horizonte, Brazil. Rio de Janeiro: ABCM; 2008.

33. Kuan Y-D and El-Gizawy AS. Numerical characterization of mold injection in resin transfer molding process. Advances in Polymer Technology. 2000; 19(3):173-179. http://dx.doi.org/10.1002/10982329(200023)19:3\%3C173::AID-ADV2\%3E3.0.CO;2-C

34. Shin KS, Song YS and Youn JR. Radial flow advancement in multi-layered preform for resin transfer molding. Korea-Australia Rheology Journal. 2006; 18(4):217-224. 